\title{
Fuzzy implicators related to (quasi-)copulas
}

\author{
Anna Kolesárová ${ }^{a}$ and Radko Mesiar ${ }^{b, c}$ \\ ${ }^{a}$ Institute of Information Engineering, Automation and Mathematics, \\ Fac. of Chemical and Food Technology, Slovak University of Technology in Bratislava, \\ Radlinského 9, 81237 Bratislava Slovakia, \\ anna.kolesarova@stuba.sk \\ ${ }^{b}$ Department of Mathematics and Descriptive Geometry, \\ Fac. of Civil Engineering, Slovak University of Technology in Bratislava, \\ Radlinského 11, 81005 Bratislava, Slovakia, \\ ${ }^{c}$ University of Ostrava, \\ Institute for Research and Applications of Fuzzy Modelling, \\ 30. dubna 22, Ostrava, Czech Republic, \\ radko.mesiar@stuba.sk
}

\begin{abstract}
In this paper, we study relations between fuzzy implicators and some kinds of fuzzy conjunctors, in particular, quasi-copulas and copulas. We show that there is a duality between the classes of all quasi-copulas and 1Lipschitz fuzzy implicators. In addition, we introduce a construction method for fuzzy implicators based on any two copulas and a Lebesgue integrable fuzzy implicator, and discuss some special cases as well.
\end{abstract}

Keywords: Copula, Fuzzy conjunctor, Fuzzy implicator, Quasi-copula.

\section{Introduction}

Boolean functions $\{0,1\}^{2} \rightarrow\{0,1\}$ determining truth values of the Boolean negation, conjunction, disjunction and implication, denoted below by $n, A N D$, $O R$ and $I M P$, respectively, are linked by the rules excluding their independence. For example, for all $x, y \in\{0,1\}$ we have

$$
\begin{aligned}
n(x) & =\operatorname{IMP}(x, 0)=1-x \\
\operatorname{AND}(x, y) & =1-\operatorname{IMP}(x, 1-y) \\
\operatorname{OR}(x, y) & =1-\operatorname{IMP}(1-x, y)
\end{aligned}
$$

etc. Any sound extension of the Boolean logical operators mentioned above, should extend the relations given in (1) - (3). This is, in particular, the case of fuzzy logical operators, where the Boolean range $\{0,1\}$ of the truth values have been extended into the real unit interval $[0,1]$, see, e.g., $[7,17]$. As a general approach to the axiomatic characterization of fuzzy logical operators one can consider a coordinate-wise extension of the corresponding Boolean operators. Let us note that as far as terminology is concerned, it is not unified, and in this paper, fuzzy extensions of the Boolean operators $A N D, O R, I M P$ and $n$ will be called fuzzy conjunctors, disjunctors, implicators, and fuzzy negators, respectively (similarly to, e.g., [4, 5]). The main aim of our contribution is to study some new constructions of fuzzy implicators and their connections with particular fuzzy conjunctors. To avoid superfluous repetitions of the well-known notions, in this paper, we only recall the notions necessary for developing the intended topic.

Definition 1.1. A function $C O N:[0,1]^{2} \rightarrow[0,1]$ is called a fuzzy conjunctor if it is monotone, 0 is its annihilator and 1 an idempotent element (i.e., $\operatorname{CON}(x, 0)=\operatorname{CON}(0, x)=0$ for each $x \in[0,1]$ and $\operatorname{CON}(1,1)=1)$.

The class of all fuzzy conjunctors will be denoted by $\mathcal{C O N}$.

Obviously, a function $C O N:[0,1]^{2} \rightarrow[0,1]$ is a fuzzy conjunctor if and only if it is a monotone extension of the Boolean conjunction operator $A N D$, $\left.C O N\right|_{\{0,1\}^{2}}=A N D$. Though Zadeh in [17] originally proposed as a fuzzy conjunctor the minimum operator, there were also mentioned some other possible fuzzy conjunctors, such as the product $\Pi$, or a partial sum $x+y-1$ defined for all $x, y \in[0,1]$ satisfying $x+y \geq 1$. This partial sum is related to the fuzzy conjunctor $W:[0,1]^{2} \rightarrow[0,1]$ given by $W(x, y)=\max \{0, x+y-1\}$. Note that in the framework of t-norms, $W$ is called the Eukasiewicz t-norm and usually denoted by $T_{L}[9]$, in the framework of copulas $W$ is called the Fréchet-Hoeffding lower bound [13].

In this contribution we will work with particular subclasses of fuzzy conjunctors only, namely, with the classes of all copulas $\mathcal{C}$ and quasi-copulas $\mathcal{Q}$.

Definition 1.2. A fuzzy conjunctor $F:[0,1]^{2} \rightarrow[0,1]$ is a copula, if

(i) $e=1$ is a neutral element of $F$, i.e., $F(x, 1)=$ $F(1, x)=x$ for each $x \in[0,1]$; 
(ii) F is 2-increasing, i.e.,

$$
F\left(x^{\prime}, y^{\prime}\right)-F\left(x^{\prime}, y\right) \geq F\left(x, y^{\prime}\right)-F(x, y)
$$

for all $x, x^{\prime}, y, y^{\prime} \in[0,1]$ with $x \leq x^{\prime}, y \leq y^{\prime}$.

Definition 1.3. A fuzzy conjunctor $F:[0,1]^{2} \rightarrow[0,1]$ is a quasi-copula, if

(i) $e=1$ is a neutral element of $F$;

(ii) F is 1-Lipschitz, i.e.,

$$
\left|F\left(x^{\prime}, y^{\prime}\right)-F(x, y)\right| \leq\left|x^{\prime}-x\right|+\left|y^{\prime}-y\right|
$$

for all $x, x^{\prime}, y, y^{\prime} \in[0,1]$.

Note that each copula satisfies the 1-Lipschitz property, thus it is also a quasi-copula. In general, we have $\mathcal{C} \varsubsetneqq \mathcal{Q} \varsubsetneqq \mathcal{C O N}$.

Example 1.1. Consider any $a \in[0,1]$.

(i) Let $F_{a}:[0,1]^{2} \rightarrow[0,1]$ be given by $F_{a}(x, y)=$ $\operatorname{med}\{\min \{x, y\}, a, x+y-1\}$, where med denotes the classical median. Then $F_{a}$ is a copula, $F_{0}=W$, $F_{1}=$ Min .

(ii) If $F_{a}$ is given by $F_{a}(x, y)=\operatorname{med}\{x y, a, x+y-1\}$, then $F_{a}$ is for each $\left.a \in\right] 0,1$ [ a proper quasi-copula, i.e., $F_{a} \in \mathcal{Q} \backslash \mathcal{C}$.

Note that throughout the paper, from the class of all fuzzy negators, we will only use the standard fuzzy negator introduced by Zadeh in [17], i.e., $N_{s}:[0,1] \rightarrow$ $[0,1], N_{s}(x)=1-x$

Definition 1.4. A function $I:[0,1]^{2} \rightarrow[0,1]$ is called a fuzzy implicator if:

(i) I is decreasing in the first variable and increasing in the second one;

(ii) $I(0,0)=I(1,1)=1$ and $I(1,0)=0$.

We denote the class of all fuzzy implicators by $\mathcal{I}$. From the hybrid monotonicity of fuzzy implicators it can be deduced that for each $I \in \mathcal{I}$ and each $x \in[0,1]$ we have $I(0, x)=1$ and $I(x, 1)=1$. Each fuzzy implicator $I$ is a coordinate-wise monotone extension of the Boolean implication operator $I M P,\left.I\right|_{\{0,1\}^{2}}=I M P$.

Some other properties of fuzzy implicators which are often of interest, are the following ones:

- The left neutrality principle,

$$
I(1, y)=y, \quad y \in[0,1] .
$$

- The identity principle,

$$
I(x, x)=1, \quad x \in[0,1] .
$$

- The ordering property,

$$
x \leq y \Leftrightarrow I(x, y)=1, \quad x, y \in[0,1]
$$

- The law of contraposition (with respect to the standard fuzzy negator)

$$
I(1-y, 1-x)=I(x, y), \quad x, y \in[0,1] .
$$

- The exchange principle,

$$
I(x, I(y, z))=I(y, I(x, z)), \quad x, y, z \in[0,1] .
$$

Let $I$ be a fuzzy implicator. If for each $x \in[0,1]$, $I(x, 0)=N_{s}(x)$, we will say that $N_{s}$ is the natural negator corresponding to $I$.

Finally, let us mention several typical fuzzy implicators which will be used throughout the paper.

Example 1.2. The following $[0,1]^{2} \rightarrow[0,1]$ functions are examples of fuzzy implicators:

- the Eukasiewicz fuzzy implicator $I_{L}, I_{L}(x, y)=$ $\min \{1,1-x+y\}$

- the Reichenbach fuzzy implicator $I_{R}, I_{R}(x, y)=$ $1-x+x y$;

- the Kleene-Dienes fuzzy implicator, $I_{K D}(x, y)=$ $\max \{1-x, y\}$;

- the Gödel fuzzy implicator,

$$
I_{G}(x, y)= \begin{cases}1 & \text { if } x \leq y \\ y & \text { otherwise }\end{cases}
$$

- the Goguen fuzzy implicator,

$$
I_{G G}(x, y)= \begin{cases}1 & \text { if } x \leq y \\ \frac{y}{x} & \text { otherwise. }\end{cases}
$$

\section{$2 \quad$ Fuzzy implicators and quasi-copulas}

Observe that the relation between Boolean conjunction operator $A N D$ and the Boolean implication operator $I M P$ given in (2), is also preserved in the case of their fuzzy extensions $C O N$ and $I$, see $[12,14]$.

Proposition 2.1. For all $C O N \in \mathcal{C O N}$ and $I \in \mathcal{I}$ we have:

(i) $I_{C O N}:[0,1]^{2} \rightarrow[0,1]$, given by

$$
I_{C O N}(x, y)=1-C O N(x, 1-y),
$$

is a fuzzy implicator. 
(ii) $C O N_{I}:[0,1]^{2} \rightarrow[0,1]$, given by

$$
\operatorname{CON}_{I}(x, y)=1-I(x, 1-y),
$$

is a fuzzy conjunctor.

(iii) $C O N_{I_{C O N}}=C O N$ and $I_{C O N_{I}}=I$.

If $C O N \in \mathcal{C O N}$ then $I_{C O N} \in \mathcal{I}$, and vice-versa, $I \in$ $\mathcal{I}$ implies that $C O N_{I} \in \mathcal{C O N}$. Due to Proposition 2.1 , there is a duality between classes $\mathcal{C O N}$ and $\mathcal{I}$, expressed by Eqs. (6) and (7). This duality can also be studied for particular subclasses of $\mathcal{C O N}$ and $\mathcal{I}$. For example, focusing on the class of all quasi-copulas, we obtain the following characterization of the subclass $\mathcal{Q} \subset \mathcal{C O N}$.

Lemma 2.1. Let $C O N \in \mathcal{C O N}$. Then the following are equivalent.

(i) $C O N \in \mathcal{Q}$.

(ii) $C O N$ is 1-Lipschitz.

Proof: While (i) $\Rightarrow$ (ii) is a trivial consequence of the definition of a quasi-copula, for proving (ii) $\Rightarrow$ (i) we have to show that $e=1$ is a neutral element of $C O N$. This fact follows from the 1-Lipschitz property of the considered $C O N$ and the boundary conditions valid for any $C O N$. Namely, $\operatorname{CON}(1,1)-\operatorname{CON}(1, x) \leq 1-x$ implies $C O N(1, x) \geq x$, and $C O N(1, x)-C O N(1,0) \leq x$ implies $\operatorname{CON}(1, x) \leq x$, i.e., $\operatorname{CON}(1, x)=x$ for each $x \in[0,1]$. A similar proof can be done for the property $C O N(x, 1)=x$ for each $x \in[0,1]$.

If we denote by $\mathcal{I}_{1-\text { Lip }}$ the class of all 1-Lipschitz fuzzy implicators, we can formulate the following interesting result.

Theorem 2.1. Let $I \in \mathcal{I}$. Then the following are equivalent.

(i) $I \in \mathcal{I}_{1-\text { Lip }}$.

(ii) $C O N_{I} \in \mathcal{Q}$.

Proof: (i) $\Rightarrow$ (ii): Let $I \in \mathcal{I}_{1-\text { Lip. }}$ Then, due to the 1-Lipschitz property and the boundary conditions $I(1,1)=1$ and $I(1,0)=0$, for each $y \in[0,1]$ we obtain $I(1, y)=I(1, y)-I(1,0) \leq y$ and $1-I(1, y)=$ $I(1,1)-I(1, y) \leq 1-y$, which yields $I(1, y) \geq y$, and thus, we have $I(1, y)=y$, i.e., $I$ satisfies the left neutrality principle (NP).

Similarly, we can show that for each $x \in[0,1]$ we have $I(x, 0)=1-x$. Therefore, for $Q=C O N_{I}$ we can write $Q(1, y)=1-I(1,1-y)=1-(1-y)=y$ for each $y \in[0,1]$, and similarly, $Q(x, 1)=1-I(x, 0)=$ $1-(1-x)=x$ for all $x \in[0,1]$, which means that $e=1$ is a neutral element of $Q$.

Moreover, for all $x, x^{\prime}, y, y^{\prime} \in[0,1]$ we have

$$
\begin{aligned}
\mid Q\left(x^{\prime}, y^{\prime}\right) & -Q(x, y) \mid \\
& =\left|1-I\left(x^{\prime}, 1-y^{\prime}\right)-1+I(x, 1-y)\right| \\
& \leq\left|x^{\prime}-x\right|+\left|y^{\prime}-y\right|,
\end{aligned}
$$

i.e., $Q$ is also 1-Lipschitz, thus a quasi-copula.

Similarly, one can also show the validity of (ii) $\Rightarrow$ (i). We omit the details.

Theorem 2.1 shows a duality between the subclasses of 1-Lipschitz fuzzy implicators and 1-Lipschitz fuzzy conjunctors. If we considered some additional properties of fuzzy implicators, we should obtain more specific subclasses of fuzzy conjunctors. Have a look at the following examples:

Example 2.1. Let $I \in \mathcal{I}_{1-\text { Lip }}$ satisfy the ordering property ( $\mathbf{O P})$. Then the related quasi-copula $Q=$ $C O N_{I}$ satisfies the equality $Q(x, y)=0$ if and only if $x \leq 1-y$. The only quasi-copula satisfying this property is $Q=W$, and thus

$I(x, y)=1-W(x, 1-y)=\min \{1,1-x+y\}=I_{L}(x, y)$

for each $(x, y) \in[0,1]^{2}$, and we can conclude that:

The only 1-Lipschitz fuzzy implicator satisfying the ordering property (OP) is the Eukasiewicz one.

Example 2.2. Let $I \in \mathcal{I}_{1-\text { Lip }}$ satisfy the exchange principle (EP) and the law of contraposition (CP). These properties of I are equivalent to the associativity of the corresponding quasi-copula $Q=C O N_{I}$, i.e., $Q$ is a 1-Lipschitz triangular norm, or equivalently, associative copula $[9,16]$. Thus $Q=\left(\left\langle a_{k}, b_{k}, f_{k}\right\rangle\right)$ $k \in \mathcal{K})$ is an ordinal sum copula,

$Q(x, y)= \begin{cases}f_{k}^{-1}\left(\min \left\{f_{k}\left(a_{k}\right), f_{k}(x)+f_{k}(y)\right\}\right) \\ & \left.\text { if }(x, y) \in] a_{k}, b_{k}\right]^{2}, k \in \mathcal{K} \\ \min \{x, y\} & \text { otherwise, }\end{cases}$

where (]$a_{k}, b_{k}[\mid k \in \mathcal{K})$ is a system of disjoint open subintervals of $[0,1]$ (possibly empty), and for each $k \in$ $\mathcal{K}, f_{k}:\left[a_{k}, b_{k}\right] \rightarrow[0, \infty]$ is a continuous convex strictly decreasing function with $f_{k}\left(b_{k}\right)=0$.

Consequently,

$I(x, y)=\left\{\begin{array}{c}1-f_{k}^{-1}\left(\min \left\{f_{k}\left(a_{k}\right), f_{k}(x)+f_{k}(1-y)\right\}\right) \\ \left.\text { if }(x, 1-y) \in] a_{k}, b_{k}\right]^{2}, k \in \mathcal{K} \\ I_{K D}(x, y) \quad \text { otherwise. }\end{array}\right.$

In particular, if we consider $\mathcal{K}=\{1\},] a_{1}, b_{1}[=] 0,1[$ and $f_{1}(x)=\frac{1}{x}-1$, for all $(x, y) \neq(0,0)$ we obtain the 
function

$$
Q(x, y)=\frac{x y}{x+y-x y}
$$

which is known as a member of the Ali-Haq-Mikhail copula family, or Clayton copula family, or as a member of the Hamacher family of t-norms, see [9, 13]. The corresponding fuzzy implicator is given by

$$
I(x, y)=\frac{1-x-y+2 x y}{1-y+x y}
$$

(with convention $\frac{0}{0}=1$ ).

Note that there are also some other relations between quasi-copulas and fuzzy implicators, for example, if they are related by Galois connections (adjointness or residuation property). More details about these relations can be found in $[5,8]$.

\section{$3 \quad$ Fuzzy implicators and copulas}

We briefly mention some recent constructions of fuzzy implicators by means of copulas and relations between them. Besides the relations between copulas and fuzzy implicators based on Galois connections discussed in [15], several other connections between them were studied, e.g., in $[2,6]$. For example, Grzegorzewski in [6] studied construction of fuzzy implicators by means of copulas given by the formula $I_{C}(x, y)=\frac{C(x, y)}{x}(x>0)$, valid for a subclass of copulas satisfying the inequality $C\left(x^{\prime}, y\right) \cdot x \leq C(x, y) \cdot x^{\prime}$ for all $x<x^{\prime}$ and $y \in[0,1]$. Note that copulas Min and $\Pi$ satisfy the given constraint, and result in $I_{M i n}=I_{G G}$ and $I_{\Pi}=I_{G}$, but this approach is not appropriate for $W, I_{W}$ is not a fuzzy implicator. From approaches discussed in [2], we recall a construction yielding for any copula $C$ the function $I^{C}:[0,1]^{2} \rightarrow[0,1], I^{C}(x, y)=1-x+C(x, y)$, which belongs to $\mathcal{I}_{1-\text { Lip }}$. We have $I^{M i n}=I_{L}, I^{\Pi}=I_{R}$, $I^{W}=I_{K D}$. Some other constructions of copulas by means of fuzzy implicators (and vice-versa) have also been studied, e.g., in [10, 11].

Now, motivated by the so-called Darsow product of copulas $*: \mathcal{C} \times \mathcal{C} \rightarrow \mathcal{C}$ introduced and studied in [3], we propose a new construction method for fuzzy implicators based on a fixed $I \in \mathcal{I}$ and a couple of copulas $C_{1}, C_{2} \in \mathcal{C}$.

Theorem 3.1. Let $I \in \mathcal{I}$ be a Lebesgue integrable function, and $C_{1}, C_{2} \in \mathcal{C}$. Then the function $J_{I, C_{1}, C_{2}}:[0,1]^{2} \rightarrow[0,1]$, given by

$$
J_{I, C_{1}, C_{2}}(x, y)=\int_{0}^{1} I\left(f_{x}(t), g_{y}(t)\right) \mathrm{d} t
$$

where

$$
f_{x}(t)= \begin{cases}0 & \text { if } \frac{\partial C_{1}(x, t)}{\partial t} \text { does not exist } \\ \frac{\partial C_{1}(x, t)}{\partial t} & \text { otherwise, }\end{cases}
$$

and

$$
g_{y}(t)= \begin{cases}0 & \text { if } \frac{\partial C_{2}(t, y)}{\partial t} \text { does not exist } \\ \frac{\partial C_{2}(t, y)}{\partial t} & \text { otherwise, }\end{cases}
$$

is a fuzzy implicator, i.e., $J_{I, C_{1}, C_{2}} \in \mathcal{I}$.

Proof: We first observe that for each $t \in[0,1]$ and all $C_{1}, C_{2} \in \mathcal{C}$ we have

$$
f_{1}(t)=g_{1}(t)=1 \text { and } f_{0}(t)=g_{0}(t)=0
$$

These properties ensure the validity of the boundary conditions: $J_{I, C_{1}, C_{2}}(0,0)=J_{I, C_{1}, C_{2}}(1,1)=1$ and $J_{I, C_{1}, C_{2}}(1,0)=0$. The 2-increasing property of copulas ensures that the functions $f_{x}$ and $g_{y}$ are increasing a.e., which implies the decreasingness of $J_{I, C_{1}, C_{2}}$ in the first variable and increasingness in the second one. Summarizing, $J_{I, C_{1}, C_{2}}$ is in $\mathcal{I}$.

It is only a matter of computation to show the validity of the following consequence of Theorem 3.1.

Corollary 3.1. Let $I \in \mathcal{I}$ be a Lebesgue integrable fuzzy implicator satisfying the (NP) property, and let $N_{s}$ be its natural negator. Then for any $C_{1}, C_{2} \in \mathcal{C}$, the function $J_{I, C_{1}, C_{2}}$ given by (8) is a fuzzy implicator with natural negator $N_{s}$, satisfying the (NP) property.

Observe that if $I$ is a fuzzy implicator with natural negator $N_{s}$, satisfying the (NP) property, and such that range $\operatorname{Ran} f_{x} \subseteq\{0,1\}$ for all $x \in[0,1]$, or Ran $g_{y} \subseteq\{0,1\}$ for all $y \in[0,1]$, respectively (see Theorem 3.1), then $J_{I, C_{1}, C_{2}}$ does not depend on $I$.

Example 3.1. Consider a copula $C_{a}$ given by

$$
C_{a}(x, y)=\operatorname{med}\{0, x+a y-a, y\}
$$

(see Fig. 1), an arbitrary Lebesgue integrable fuzzy implicator $I$ satisfying $I(x, 0)=1-x$ for each $x \in$ $[0,1]$, and an arbitrary copula $C$. Then for each $y \in$ $\left[0,1\left[, g_{y}=\mathbf{1}_{] a(1-y), a+(1-a) y[}\right.\right.$ and $g_{1}=\mathbf{1}_{[0,1]}$ (see Fig. 2), and further, 


$$
\begin{aligned}
J_{I, C, C_{a}}(x, y) & \int_{0}^{a(1-y)} I\left(f_{x}(t), 0\right) \mathrm{d} t+\int_{a(1-y)}^{a+(1-a) y} I\left(f_{x}(t), 1\right) \mathrm{d} t \\
= & \int_{a+(1-a) y}^{1} I\left(f_{x}(t), 0\right) \mathrm{d} t \\
= & 1-\int_{0}^{a(1-y)} f_{x}(t) \mathrm{d} t-\int_{a+(1-a) y}^{1} f_{x}(t) \mathrm{d} t \\
= & 1-C(x, a(1-y))-C(x, 1) \\
+ & C(x, a+(1-a) y) \\
= & 1-x-C(x, a(1-y))+C(x, a+(1-a) y) .
\end{aligned}
$$

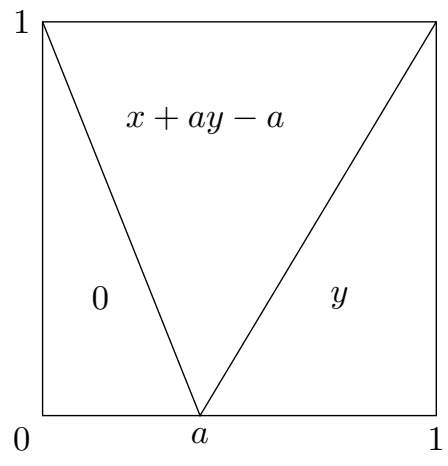

Fig. 1: Copula $C_{a}$ given in Ex. 3.1

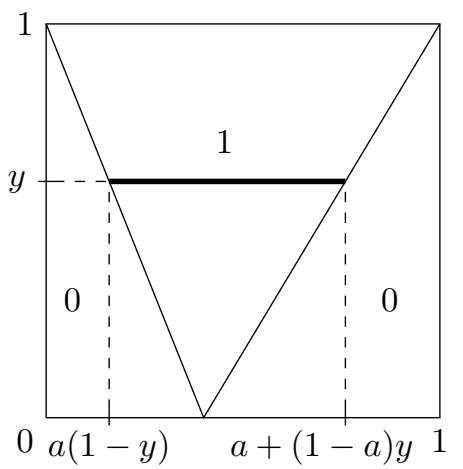

Fig. 2: The function $g_{y}=\mathbf{1}_{] a(1-y), a+(1-a) y[}$ corresponding to the copula $C_{a}$, see Th. 3.1 and Ex. 3.1

As we can see, the resulting function $J_{I, C, C_{a}}$ in Example 3.1 depends on $C \in \mathcal{C}$ and $a \in[0,1]$ only. Hence, we can introduce a new copula based construction of fuzzy implicators, assigning to each $C \in \mathcal{C}$ and any $a \in[0,1]$ directly a fuzzy implicator $I_{a, C}=J_{I, C, C_{a}}$.

Proposition 3.1. Let $C$ be any copula and $a \in[0,1]$. Then the function $I_{a, C}:[0,1]^{2} \rightarrow[0,1]$ given by

$I_{a, C}(x, y)=1-x-C(x, a(1-y))+C(x, a+(1-a) y)$ is a fuzzy implicator.
Note that for each $C \in \mathcal{C}, I_{0, C}(x, y)=1-x+C(x, y)$, i.e., $I_{0, C}=I^{C}$, which means that our construction $I_{a, C}$ also covers the construction $I^{C}$ from [2] mentioned above.

The other boundary case for $a=1$ also leads to a wellknown relation between copulas and fuzzy implicators, namely,

$$
I_{1, C}(x, y)=1-C(x, 1-y) .
$$

Finally, for each $(x, y) \in[0,1]^{2}$ we have

$$
I_{0.5, C}(x, y)=1-x-C\left(x, \frac{1-y}{2}\right)+C\left(x, \frac{1+y}{2}\right) .
$$

\section{Conclusion}

We have discussed the relations between fuzzy implicators and some particular classes of fuzzy conjunctors, namely the classes of quasi-copulas and copulas. We have shown the existence of a duality between the class of all quasi-copulas and the class of 1-Lipschitz fuzzy implicators. In Theorem 3.1, we have introduced a powerful tool for constructing fuzzy implicators based on a Lebesgue integrable fuzzy implicator $I$ and any two copulas $C_{1}, C_{2}$. Moreover, some particular cases were exemplified. We believe that our approach opens a new look at constructions of fuzzy implicators. In particular, we expect that Theorem 3.1 can be further generalized, considering more general systems of unary functions $\left(f_{x}\right)_{x \in[0,1]},\left(g_{y}\right)_{y \in[0,1]}$.

\section{Acknowledgement}

The authors are grateful for the support of the projects VEGA $1 / 0614 / 18$ and VEGA 1/0006/19. Moreover, the work of R. Mesiar on this paper was supported by the NPUII project LQ1602 and the project APVV$17-0066$.

\section{References}

[1] M. Baczyński, B. Jayaram, Fuzzy Implications. Studies in Fuzziness and Soft Computing Series, 231, Springer-Verlag, Heidelberg, 2008.

[2] M. Baczyński, P. Grzegorzewski, R. Mesiar, P. Helbin, and W. Niemyska, Fuzzy implications based on semicopulas. Fuzzy Sets and Systems 323 (2017) 138-151.

[3] W. F. Darsow, B. Nguyen, and E. T. Olsen, Copulas and Markov processes. Illinois J. Math. 36(4) (1992) 600-642.

[4] B. De Baets, Coimplicators, the forgotten connectives. Tatra Mt. Math. Publ. 12 (1997) 229-240. 
[5] F. Durante, E. P. Klement, R. Mesiar, and C. Sempi, Conjunctors and their residual implicators: Characterizations and construction methods. Mediterranean J. Math. 4 (2007) 343-356.

[6] P. Grzegorzewski, Probabilistic implications. Fuzzy Sets and Systems 226 (2013) 53-66.

[7] P. Hájek, Metamathematics of Fuzzy Logic. Kluwer, Dordrecht, 1998.

[8] P. Hájek, R. Mesiar, On copulas, quasi-copulas and fuzzy logic. Soft Computing 12 (2008) 1239 1243 .

[9] E. P. Klement, R. Mesiar, and E. Pap, Triangular Norms. Kluwer Academic Publishers, Dordrecht, 2000 .

[10] S. Massanet, D. Ruiz-Aguilera, and J. Torrens, On two construction methods of copulas from fuzzy implication functions. Progress in AI 5(1) (2016) 1-14.

[11] S. Massanet, A. Pradera, D. Ruiz-Aguilera, and J. Torrens, From three to one: Equivalence and characterization of material implications derived from co-copulas, probabilistic $S$-implications and survival $S$-implications. Fuzzy Sets and Systems 323 (2017) 103-116.

[12] R. Mesiar, A. Kolesárová, H. Bustince, and J. Fernandez, Dualities in the class of extended Boolean functions. Fuzzy Sets and Systems 332 (2018) 78 92.

[13] R. B. Nelsen, An Introduction to Copulas. Second ed. Lecture Notes in Statistics 139, Springer, New York, 2006.

[14] A. Pradera, G. Beliakov, H. Bustince, and B. De Baets, A review of the relationships between implication, negation and aggregation functions from the point of view of material implication. Inform. Sci. 329 (2016) 357-380.

[15] E. Sainio, E. Turunen, and R. Mesiar, A characterization of fuzzy implications generated by generalized quantifiers. Fuzzy Sets and Systems 159 (2008) 491-499.

[16] B. Schweizer, A. Sklar, Probabilistc Metric Spaces. Dover Books on Mathematics. North Holland Series in Probability and Applied Mathematics. Courier Corporation, 2005.

[17] L. A. Zadeh, Fuzzy sets. Inform. Control 8 (1965) 338-353. 